\title{
Impactos psicológicos em adultos durante a pandemia de COVID-19: uma revisão integrativa
}

\author{
Psychological impacts on adults during the COVID-19 pandemic: an integrative review \\ Impactos psicológicos en los adultos durante la pandemia del COVID-19: una revisión integrativa
}

Recebido: 21/07/2021 | Revisado: 26/07/2021 | Aceito: 26/08/2021 | Publicado: 29/08/2021

Patrícia Rayane Medeiros Castro

ORCID: https://orcid.org/0000-0002-9768-9806

Faculdade de Ensino Superior do Piauí, Brasil

E-mail: patriciarayanemedeiros@gmail.com

Sara Castro de Souza

ORCID: https://orcid.org/0000-0002-6116-5365

Faculdade de Ensino Superior do Piauí, Brasil E-mail: saracastropsi@ hotmail.com

Rodrigo Almeida Damasceno

ORCID: https://orcid.org/0000-0002-9849-113X Faculdade de Ensino Superior do Piauí, Brasil E-mail: rodrigopsico02@gmail.com

Gisele Martins do Nascimento

ORCID: https://orcid.org/0000-0002-0136-1209 Faculdade de Ensino Superior do Piaú, Brasil E-mail: giselensa@hotmail.com

Ruth Raquel Soares de Farias

ORCID: https://orcid.org/0000-0002-0988-0900 Faculdade de Ensino Superior do Piauí, Brasil E-mail: ruthraquelsf@gmail.com

\begin{abstract}
Resumo
A COVID-19, doença causada pelo novo coronavírus foi descoberta em novembro de 2019, na cidade de Wuhan, China. Com a expansão da doença e o seu caráter pandêmico, aumentaram os agravos à saúde, bem como os impactos na saúde mental da população global, gerando a necessidade de acompanhamento psicológico. O objetivo do estudo foi analisar os impactos psicológicos em adultos causados pela pandemia de COVID-19. Foi realizada uma revisão integrativa da literatura, a partir de artigos primários oriundos das bases de dados Scientific Eletronic Library Online (SCIELO) e Literatura Latino-Americana e do Caribe em Ciências da Saúde (LILACS). Foram encontrados 18 artigos relacionados ao tema. Os dados apresentados neste estudo reforçam a teoria de que a pandemia de COVID-19 provocou mudanças significativas na vida dos adultos, principalmente no que se refere aos impactos psicológicos diagnosticados, as mudanças no estilo de vida e a repercussão nos sentimentos das pessoas, bem como a autopercepção em relação à saúde mental durante a pandemia. Com o estudo, concluiu-se que os impactos psicológicos mais comuns durante a pandemia foram ansiedade, depressão e estresse. Os sentimentos mais evidentes foram medo, tristeza e preocupação com adoecimento por COVID-19, provocando alterações do sono, diminuição das atividades físicas e aumento no consumo de alimentos industrializados.
\end{abstract}

Palavras-chave: Coronavírus; Isolamento social; Pandemia; Covid-19; Saúde mental; Transtornos mentais.

\begin{abstract}
COVID-19, a disease caused by the new coronavirus, was discovered in November 2019 in the city of Wuhan, China. With the expansion of the disease and its pandemic character, the damage to health has increased, as well as the impacts on the mental health of the global population, generating the need for psychological support. The aim of the study was to analyze the psychological impacts on adults caused by the COVID-19 pandemic. An integrative literature review was carried out, based on primary articles from the Scientific Electronic Library Online (SCIELO) and Latin American and Caribbean Health Sciences (LILACS) databases. Eighteen articles related to the topic were found. The data presented in this study reinforce the theory that the COVID-19 pandemic caused significant changes in the lives of adults, especially with regard to the diagnosed psychological impacts, changes in lifestyle and the impact on people's feelings, as well as self-perception of mental health during the pandemic. The study concluded that the most common psychological impacts during the pandemic were anxiety, depression and stress. The most evident feelings were fear, sadness and concern about illness due to COVID-19, causing sleep disorders, decreased physical activity and increased consumption of processed foods.
\end{abstract}

Keywords: Coronavirus; Social isolation; Pandemic; Covid-19; Mental health; Mental disorders. 


\begin{abstract}
Resumen
COVID-19, una enfermedad causada por el nuevo coronavirus, fue descubierta en noviembre de 2019 en la ciudad de Wuhan, China. Con la expansión de la enfermedad y su carácter pandémico, se ha incrementado el daño a la salud, así como los impactos en la salud mental de la población mundial, generando la necesidad de apoyo psicológico. El objetivo del estudio fue analizar los impactos psicológicos en los adultos provocados por la pandemia de COVID-19. Se realizó una revisión integradora de la literatura, a partir de artículos primarios de las bases de datos de la Biblioteca Electrónica Científica en Línea (SCIELO) y Ciencias de la Salud de América Latina y el Caribe (LILACS). Se encontraron dieciocho artículos relacionados con el tema. Los datos presentados en este estudio refuerzan la teoría de que la pandemia COVID-19 provocó cambios significativos en la vida de los adultos, especialmente en lo que se refiere a los impactos psicológicos diagnosticados, cambios en el estilo de vida y el impacto en los sentimientos de las personas, así como la autopercepción de salud mental durante la pandemia. El estudio concluyó que los impactos psicológicos más comunes durante la pandemia fueron la ansiedad, la depresión y el estrés. Los sentimientos más evidentes fueron el miedo, la tristeza y la preocupación por la enfermedad por COVID-19, que provoca trastornos del sueño, disminución de la actividad física y aumento del consumo de alimentos procesados.
\end{abstract}

Palabras clave: Coronavirus; Aislamiento social; Pandemia; Covid-19; Salud mental; Desordenes mentale.

\title{
1. Introdução
}

A COVID-19, doença causada pelo novo coronavírus, gerou uma das maiores pandemias do mundo moderno, atingindo, hoje, quase duzentos milhões de pessoas e provocando quase quatro milhões de mortes (OMS, 2021).

Os primeiros casos de COVID-19 surgiram em novembro de 2019, na cidade de Wuhan, na China Oriental. Mas somente em 31 de dezembro, a Organização Mundial da Saúde (OMS) foi informada sobre um surto de pneumonia, na cidade, sendo em 7 de janeiro de 2020, confirmadas infecções pelo novo coronavírus (OPAS, 2021). Com a situação de contaminação entre as pessoas, há uma contínua preocupação, no Brasil e no mundo, causando estresse físico e mental das pessoas (Duan, L.; Zhu, G. 2020).

Conforme a evolução da pandemia e seus agravos à saúde, ocorre também, um impacto na saúde mental da população, evidenciado por ansiedade, medo, insegurança, bem como sensação de incapacidade. Pensamentos estressantes, intrusivos e negativos geram dificuldades em todas as dimensões da vida do indivíduo, destacando a importância de lidar com os problemas emocionais, de modo que haja o controle da propagação do vírus, através do cumprimento das medidas sanitárias impostas pelo Governo e, ainda, melhoria na saúde mental. Do ponto de vista científico, os comportamentos inadequados trazem prejuízo à saúde do indivíduo e da coletividade (Lin, E. C. L.; Peng, Y. C.; Tsai, J. C. H., 2012; Qiu et al., 2020; Wang et al., 2020).

A pandemia tem causado grande impacto social, econômico e pessoal na vida das pessoas, uma vez que o número dos que sofrem danos psicológicos passa a ser maior que o daquelas acometidas pela infecção do vírus. Caso não haja o acompanhamento necessário com psicólogos e psiquiatras, muitas dessas pessoas podem apresentar graves consequências e levarem mais tempo para solucionar problemas psicológicos do que outros agravos causados pela pandemia (Lima, 2020; Ornell et al., 2020).

O isolamento social derivado da Covid-19 desencadeou o agravamento dos quadros de pessoas que já se encontravam afetadas por doenças mentais. Já as que não eram, grande parte desenvolveu problemas psicológicos e uma série de transtornos de grande importância clínica, como ansiedade generalizada e depressão (Lima, 2020). Assim, o estado de solidão que muitas pessoas sofrem, na pandemia, permite o desenvolvimento de doenças mentais e problemas psicológicos que necessitam de acompanhamento (Donida et al., 2021).

Vale ressaltar que, num cenário pandêmico a vigilância dos agravos à saúde física, são priorizados. Com isso, a saúde mental das pessoas fica negligenciada, exibindo um quadro de pessoas, psicologicamente afetadas, além dos prejuízos à vida social, econômica e pessoal. Há uma grande necessidade de prestar assistências às pessoas, no atual período de pandemia, de modo que o apoio prestado por profissionais de saúde e cuidadores produza indicadores positivos e, não obstante, promova medidas preventivas a doenças mentais (Schmidt et al., 2020). 
Evidenciar as doenças mentais e desordens psicológicas provocadas pela pandemia do novo coronavírus, abre portas para a criação de políticas públicas de atenção psicossocial que promovam o bem-estar das pessoas em situação de isolamento social. Além disso, permite que os cuidados em saúde se estendam além da saúde física, contemplando as questões mentais do ser humano e toda a complexidade psicológica permeada pela pandemia. Assim, o objetivo deste trabalho foi analisar os impactos psicológicos em adultos causados pela pandemia de COVID-19 e, especificamente, identificar os principais impactos psicológicos que interferem na vida das pessoas adultas, apontar as mudanças no estilo de vida e nos sentimentos das pessoas, e descrever a autopercepção de pessoas em relação à sua saúde mental.

\section{Metodologia}

Trata-se de uma revisão integrativa da literatura, que segundo Souza, Silva e Carvalho (2010) permite uma abordagem ampla de um determinado tema, de modo que o pesquisador utilize estudos experimentais e não-experimentais, tornando consistente e claro o entendimento dos leitores, através da apresentação de resultados elucidados pela literatura.

A coleta de dados foi realizada em março de 2021, utilizando-se como bases de dados as bibliotecas digitais Scientific Eletronic Library Online (SCIELO) e Literatura Latino-Americana e do Caribe em Ciências da Saúde (LILACS). Como Descritores em Ciências da Saúde (DeCS), foram utilizados na busca: coronavírus; isolamento social; pandemia; saúde mental e; transtornos mentais. Os critérios de inclusão adotados para a realização da pesquisa foram: artigos originais de dados primários, publicados nos idiomas português, inglês e espanhol. Foram excluídos do estudo, artigos de revisão, editoriais, cartas abertas, artigos duplicados, artigos com resultados inconclusos, dissertações, monografias e teses.

A busca aconteceu em duas fases, cruzando os descritores por meio do operador booleano "AND", com os títulos em inglês. Foi obedecido o seguinte cruzamento: Coronavírus and Mental Disorders; Pandemic and Mental Health e; Social Isolation and Mental Disorders. Esse método de busca permite a condensação de artigos publicados em diferentes periódicos nacionais e internacionais.

Para a realização da pesquisa não foi estabelecido período de publicação, tendo em vista que se trata de um tema atual, o que garante estudos publicados a partir de 2020. Dessa forma, o processo de busca está descrito de forma dinâmica em fluxograma, o qual exibe as etapas de seleção de estudos elegíveis à revisão de literatura. 
Fluxograma 1 - Processo de seleção de artigos elegíveis à revisão
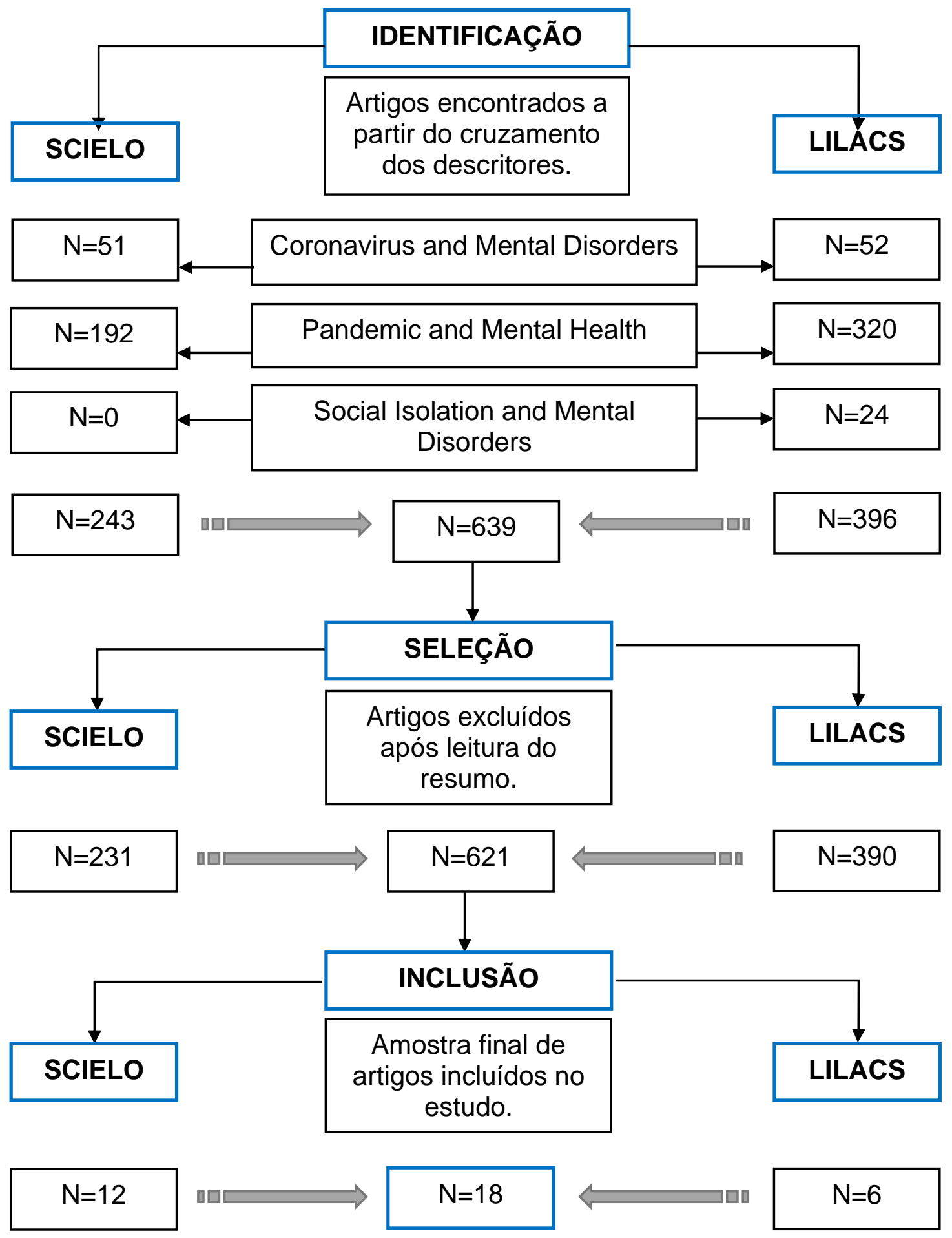

Fonte: Dados da pesquisa.

\section{Resultados}

O estudo resultou em 18 artigos encontrados, dos quais 12 (66,65\%) oriundos da SCIELO e 6 (33,35\%) da LILACS. O Quadro 1, apresenta os dados de cada artigo, conforme o título, autor/ano, delineamento da pesquisa e objetivos. 
Quadro 1 - Artigos incorporados à revisão integrativa, publicados em 2020 e 2021.

\begin{tabular}{|c|c|c|c|}
\hline TÍTULO & AUTOR/ ANO & DELINEAMENTO & OBJETIVOS \\
\hline $\begin{array}{l}\text { Bienestar emocional, psicológico y } \\
\text { social em adultos argentinos en } \\
\text { contexto de pandemia por COVID-19 }\end{array}$ & $\begin{array}{l}\text { Eidman et al. } \\
(2020)\end{array}$ & $\begin{array}{l}\text { Estudo transversal, de } \\
\text { caráter descritivo. }\end{array}$ & $\begin{array}{l}\text { Determinar o bem-estar emocional, psicológico e } \\
\text { social em adultos argentinos no contexto da pandemia } \\
\text { coVID- } 19 \text {. }\end{array}$ \\
\hline $\begin{array}{l}\text { COVID-19 e os impactos na saúde } \\
\text { mental: uma amostra do Rio Grande do } \\
\text { Sul, Brasil }\end{array}$ & $\begin{array}{l}\text { Duarte et al., } \\
(2020)\end{array}$ & $\begin{array}{l}\text { Estudo transversal, de } \\
\text { caráter exploratório. }\end{array}$ & $\begin{array}{l}\text { Verificar os fatores associados a indicadores de } \\
\text { sintomas de transtornos mentais em residentes do Rio } \\
\text { Grande do Sul, durante o período inicial da política de } \\
\text { distanciamento social decorrente da pandemia da } \\
\text { COVID-19. }\end{array}$ \\
\hline $\begin{array}{l}\text { COVID-19 nas prisões: efeitos da } \\
\text { pandemia sobre a saúde mental de } \\
\text { mulheres privadas de liberdade }\end{array}$ & $\begin{array}{l}\text { Santos et al. } \\
(2020)\end{array}$ & $\begin{array}{l}\text { Estudo transversal, de } \\
\text { caráter exploratório. }\end{array}$ & $\begin{array}{l}\text { Investigar o autorrelato de sintomas de ansiedade } \\
\text { relacionados à Covid-19 entre mulheres encarceradas. }\end{array}$ \\
\hline $\begin{array}{l}\text { Covid-19 no Brasil: estresse como } \\
\text { preditor da depressão }\end{array}$ & Silva, (2020) & $\begin{array}{l}\text { Estudo transversal, de } \\
\text { caráter exploratório. }\end{array}$ & $\begin{array}{l}\text { Analisar os níveis de estresse e depressão dos } \\
\text { brasileiros, bem como estabelecer um modelo } \\
\text { preditivo entre os construtos. }\end{array}$ \\
\hline $\begin{array}{l}\text { COVID-19 pandemic decrease men's } \\
\text { mental health: background and } \\
\text { consequence analysis }\end{array}$ & Sousa et al., (2021) & $\begin{array}{l}\text { Estudo observacional, } \\
\text { de caráter descritivo. }\end{array}$ & $\begin{array}{l}\text { Analisar, na perspectiva do autorreconheciente de } \\
\text { antecedentes e consequências, como a pandemia } \\
\text { COVID-19 diminui a saúde dos homens residentes no } \\
\text { Brasil. }\end{array}$ \\
\hline $\begin{array}{l}\text { COVID-19 y su asociación con } \\
\text { síntomas depresivos en población } \\
\text { española }\end{array}$ & Casas et al., (2020) & $\begin{array}{l}\text { Estudo de corte } \\
\text { transversal, de caráter } \\
\text { descritivo. }\end{array}$ & $\begin{array}{l}\text { Avaliar o efeito do confinamento sobre o humor } \\
\text { depressivo na população que vive na Espanha acima } \\
\text { de } 18 \text { anos durante o estado de alarme causado pela } \\
\text { pandemia COVID-19, analisando ainda mais a } \\
\text { possível incidência de sexo variável, idade, tamanho } \\
\text { da moradia e renda econômica. }\end{array}$ \\
\hline $\begin{array}{l}\text { Depresión y ansiedad durante el } \\
\text { aislamiento obligatorio por el COVID- } \\
19 \text { en Lima Metropolitana }\end{array}$ & $\begin{array}{l}\text { Prieto-Molinari et } \\
\text { al., (2020) }\end{array}$ & $\begin{array}{l}\text { Estudo transversal, de } \\
\text { caráter descritivo. }\end{array}$ & $\begin{array}{l}\text { Descrever a prevalência de sintomas de ansiedade e } \\
\text { depressão em adultos na Região Metropolitana de } \\
\text { Lima, durante o período de isolamento social em } \\
\text { resposta à pandemia COVID- } 19 \text {. }\end{array}$ \\
\hline $\begin{array}{l}\text { Distanciamento social, sentimento de } \\
\text { tristeza e estilos de vidada população } \\
\text { brasileira durante a pandemia de } \\
\text { COVID-19 }\end{array}$ & Malta et al., (2020) & $\begin{array}{l}\text { Estudo transversal, de } \\
\text { caráter exploratório. }\end{array}$ & $\begin{array}{l}\text { Analisar a adesão ao distanciamento social, as } \\
\text { repercussões no estado de ânimo e mudanças nos } \\
\text { estilos de vida da população adulta brasileira durante o } \\
\text { início da pandemia da COVID- } 19 \text {. }\end{array}$ \\
\hline $\begin{array}{l}\text { Efectos del confinamiento social, } \\
\text { preventivo y obligatorio sobre la salud } \\
\text { física y psíquica de los comodorenses }\end{array}$ & $\begin{array}{l}\text { Pereira; Espín; } \\
\text { Tögel, (2021) }\end{array}$ & $\begin{array}{l}\text { Estudo transversal, de } \\
\text { caráter descritivo. }\end{array}$ & $\begin{array}{l}\text { Descrever alguns dos efeitos autopercebidos pela } \\
\text { população de Comodoro sobre sua saúde física e } \\
\text { mental. }\end{array}$ \\
\hline $\begin{array}{l}\text { Emociones, preocupaciones y } \\
\text { reflexiones frente a la pandemia del } \\
\text { COVID-19 en Argentina }\end{array}$ & $\begin{array}{l}\text { Johnson; Saletti- } \\
\text { Cuesta; Tumas, } \\
\quad \text { (2020) }\end{array}$ & $\begin{array}{l}\text { Estudo de corte } \\
\text { cruzado, de caráter } \\
\text { descritivo. }\end{array}$ & $\begin{array}{c}\text { Explorar os sentimentos e expectativas gerados pelo } \\
\text { COVID-19 na Argentina durante a primeira etapa da } \\
\text { pandemia. }\end{array}$ \\
\hline $\begin{array}{l}\text { Estrés percibido asociado a la pandemia } \\
\text { por COVID-19 en la ciudad de } \\
\text { Guayaquil, Ecuador }\end{array}$ & $\begin{array}{l}\text { Yanez et al., } \\
\quad(2021)\end{array}$ & $\begin{array}{l}\text { Estudo transversal, de } \\
\text { caráter descritivo. }\end{array}$ & $\begin{array}{c}\text { Identificar os níveis de estresse associados à pandemia } \\
\text { de COVID-19, na população. }\end{array}$ \\
\hline $\begin{array}{l}\text { Factors affecting Brazilians' self-rated } \\
\text { health during the COVID-19 pandemic }\end{array}$ & $\begin{array}{l}\text { Szwarcwald et al., } \\
\qquad \text { (2021) }\end{array}$ & $\begin{array}{l}\text { Estudo transversal, de } \\
\text { caráter exploratório. }\end{array}$ & $\begin{array}{l}\text { Investigar fatores que afetam a autopercepção de saúde } \\
\text { dos brasileiros durante a pandemia da COVID-19. }\end{array}$ \\
\hline $\begin{array}{l}\text { Factors associated with stress, anxiety, } \\
\text { and depression during social distancing } \\
\text { in Brazil }\end{array}$ & Souza et al., (2021) & $\begin{array}{l}\text { Estudo transversal, de } \\
\text { caráter descritivo. }\end{array}$ & $\begin{array}{c}\text { Estimar a prevalência de sinais clínicos e sintomas de } \\
\text { estresse grave/extremo, ansiedade e depressão, bem } \\
\text { como seus fatores associados, entre os brasileiros } \\
\text { durante o distanciamento social. }\end{array}$ \\
\hline
\end{tabular}




\begin{tabular}{|c|c|c|c|}
\hline $\begin{array}{l}\text { Impacto psicosocial de la pandemia por } \\
\text { COVID-19 en adultos de Buenos Aires }\end{array}$ & $\begin{array}{l}\text { Herrera-Paz et al., } \\
\qquad(2020)\end{array}$ & $\begin{array}{l}\text { Estudo observacional, } \\
\text { de caráter descritivo. }\end{array}$ & $\begin{array}{c}\text { Analisar o impacto do isolamento social sobre hábitos } \\
\text { saudáveis e psicossociais e comportamentais, aspectos } \\
\text { durante o confinamento e restrições impostas pela } \\
\text { pandemia na região metropolitana de Buenos Aires }\end{array}$ \\
\hline $\begin{array}{l}\text { Impacto psicossocial do isolamento } \\
\text { durante pandemia de covid-19 na } \\
\text { população brasileira: análise transversal } \\
\text { preliminar }\end{array}$ & $\begin{array}{l}\text { Bezerra et al., } \\
\quad(2020)\end{array}$ & $\begin{array}{l}\text { Estudo transversal, de } \\
\text { caráter descritivo. }\end{array}$ & $\begin{array}{l}\text { Identificar preditores de estresse psicossocial com } \\
\text { dados recolhidos por questionário on-line nas redes } \\
\text { sociais em abril de } 2020 \text {. }\end{array}$ \\
\hline $\begin{array}{l}\text { Impacto sobre la salud mental durante } \\
\text { la pandemia COVID } 19 \text { en Paraguay }\end{array}$ & $\begin{array}{l}\text { Gould; Diaz; } \\
\text { Vargas, (2020) }\end{array}$ & $\begin{array}{l}\text { Estudo de corte } \\
\text { prospectivo. }\end{array}$ & $\begin{array}{l}\text { Descrever a frequência da depressão, ansiedade e } \\
\text { isônia durante a quarentena, na população paraguaiaa. }\end{array}$ \\
\hline $\begin{array}{c}\text { Intolerance of uncertainty and mental } \\
\text { health in Brazil during the Covid-19 } \\
\text { pandemic }\end{array}$ & $\begin{array}{l}\text { Ferreira } \text { et al., } \\
\quad(2020)\end{array}$ & $\begin{array}{l}\text { Estudo transversal, de } \\
\text { caráter descritivo. }\end{array}$ & $\begin{array}{l}\text { Investigar a relação entre intolerância à incerteza e } \\
\text { transtornos mentais comuns. }\end{array}$ \\
\hline $\begin{array}{l}\text { Relato de tristeza/depressão, } \\
\text { nervosismo/ansiedade e problemas de } \\
\text { sono na população adulta brasileira } \\
\text { durante a pandemia de COVID-19 }\end{array}$ & $\begin{array}{l}\text { Barros et al., } \\
\quad(2020)\end{array}$ & $\begin{array}{l}\text { Estudo transversal, de } \\
\text { caráter exploratório. }\end{array}$ & $\begin{array}{l}\text { Analisar a frequência de tristeza, nervosismo e } \\
\text { alterações do sono durante a pandemia de COVID-19 } \\
\text { no Brasil, identificando os segmentos demográficos } \\
\text { mais afetados. }\end{array}$ \\
\hline
\end{tabular}

Fonte: Dados da pesquisa.

Dos artigos apresentados, no estudo, 13 (72,22\%) foram publicados no de 2020, enquanto 5 (27,78\%) foram publicados em 2021. Desses artigos, $14(77,78 \%)$ se trata de estudos transversais.

Os dados apresentados neste estudo reforçam a teoria de que a pandemia de COVID-19 provocou mudanças significativas na vida dos adultos, principalmente no que se refere aos impactos psicológicos diagnosticados, as mudanças no estilo de vida e a repercussão nos sentimentos das pessoas, bem como a autopercepção em relação à saúde mental durante a pandemia. Duarte et al. (2020) destaca em seu estudo que, de 799 indivíduos adultos $25 \%$ relataram ter sido diagnosticado com transtorno mental. Além disso, 23,80\% faziam parte do grupo de risco e 43,40\% moravam com pessoas pertencentes ao grupo de risco para COVID-19.

Tomando como base a pesquisa de Silva (2020), com 480 pessoas, entre 18 e 65 anos, na sua maioria do sexo feminino $(77,30 \%)$, foi estabelecida uma correlação entre estresse e a predisposição à depressão. Estatisticamente, o estresse causado pela COVID-19 foi bastante relevante como preditor de depressão. Souza et al. (2021) descreve o estresse extremo, ansiedade e depressão como impactos psicológicos graves em uma população de 3.200 brasileiros maiores de 18 anos, durante a pandemia de COVID-19.

O impacto do isolamento social na vida das pessoas foi abordado por Bezerra et al. (2020), colocando o estresse familiar como um dos problemas mais evidentes durante a pandemia, em seu estudo com 3.836 participantes de 24 estados brasileiros, sendo que $87,40 \%$ mostraram-se com medo de adoecer e preocupados com o adoecimento de familiares. Além disso, $8,30 \%$ dos participantes utilizaram medicamentos porque se sentiam tristes e preocupados.

Os impactos na saúde mental durante a pandemia de COVID-19 foram descritos por Gould, Diaz e Vargas (2020), evidenciando que, de 511 participantes adultos em seu estudo, 21,30\% apresentaram ansiedade e depressão moderada, 15,90\% apresentaram ansiedade e depressão severa e que 62,50\% apresentavam algum nível de insônia relatado.

No estudo de Prieto-Molinari et al. (2020), são destacados os quadros de ansiedade e depressão em uma amostra de 565 adultos peruanos que se encontravam em estado de isolamento social, no país. Foi evidente que a população que era ativa antes da pandemia apresentou problemas psicológicos devido ao isolamento obrigatório. Semelhantemente, Yanez et al. (2021) identificou níveis elevados de estresse, numa amostra da população equatoriana durante o período de confinamento. 
Ao abordar o efeito da pandemia em 43 mulheres privadas de liberdade, na Bahia, Santos et al. (2020) relata a preocupação, o medo de adoecer e morrer, a tristeza e o nervosismo como sentimentos recorrentes entre estas, principalmente pelo desconhecimento da doença e pela situação de cárcere. Desse modo, Ferreira et al. (2020) destaca que as pessoas se sentem inseguras, tristes e ansiosas diante da incerteza da pandemia, sendo mais frequentes estes sentimentos nas mulheres.

A abordagem de Eidman et al. (2020) exibe a diferença no estado de saúde mental de homens e mulheres argentinos, onde as mulheres apresentaram níveis baixos de saúde mental em relação aos homens. Johnson, Saletti-Cuesta e Tumas (2020) demonstram que, o medo do futuro, as incertezas quanto à renda, saúde e preocupação com os familiares foram recorrentes na população argentina, principalmente nas mulheres mais velhas.

Os sentimentos vividos por adultos brasileiros durante a pandemia se expressaram com tristeza, nervosismo e ansiedade, como apontou o estudo de Barros et al. (2020). Casas et al. (2020), evidenciou através de seu estudo que, na população espanhola, mulheres apresentaram maiores sinais de depressão, ansiedade e estresse. E ainda colocando estes problemas como comuns ao período de pandemia.

Malta et al., 2020 relatou que o estilo de vida das pessoas mudou com a pandemia, com destaque aos adultos entre 18 e 39 anos, no que se refere ao consumo de bebida alcóolica e tabaco. Além disso, o estudo de Herrera-Paz et al. (2020) apontou que houve um aumento considerável do tempo à frente de tela de dispositivos, diminuição na prática de exercícios físicos e aumento do consumo de alimentos industrializados, quando entrevistados 2.912 argentinos.

Sousa et al. (2021) observou em sua pesquisa, com 200 homens brasileiros de todas as regiões do país que a pandemia agravou problemas familiares, sentimentos de fraqueza e impotência, além de afetar as relações sociais e a situação financeira.

$\mathrm{Na}$ Argentina, um inquérito realizado com 1.093 pessoas de Comodoro, apontou que a pandemia de COVID-19 desencadeou sentimentos de tristeza e medo autopercebidos, como também contribuiu para a redução drástica de atividades físicas e alimentação saudável (Pereira; Espín; Tögel, 2021). Esta autopercepção foi descrita, também, por Szwarcwald et al. (2021), num inquérito com 45.161 brasileiros, dos quais 29,40\% relataram piora na saúde durante a pandemia, sendo problemas psicológicos, dores lombares, alterações do sono e sintomas gripais.

\section{Discussão}

O presente estudo objetivou abordar aspectos relacionados aos impactos psicológicos em tempos de pandemia de COVID-19, uma vez que os adultos, por terem vida mais ativa, tem sofrido com o isolamento social. Nesse contexto, existem fortes evidências científicas de que muitas pessoas desenvolveram problemas psicológicos, além de apresentarem potencialização de problemas psicológicos, como ocorrido em uma penitenciária feminina, no estado da Paraíba que abrigava 263 detentas em 2020, sendo registrada a ocorrência de transtornos psíquicos, bem como crise de ansiedade e automutilação, os quais representam problemas agravados pela pandemia, na visão dos trabalhadores da unidade prisional (Oliveira; Rocha; Abreu, 2020).

Maia e Dias (2020) identificaram em uma amostra de 460 estudantes universitários, com idade média de 20,14 anos, aumento nos níveis de ansiedade, depressão e estresse quando comparado o período normal entre 2018 e 2019, com o período atual da pandemia. Carvalho et al. (2020) ressalta que o isolamento social causou nas pessoas um agravante estado de medo, solidão e preocupação com familiares quando analisou o comportamento de 73 brasileiros adultos, mostrando que as facetas da COVID-19 causam sentimentos prejudiciais à saúde mental.

Esteves, Oliveira e Argimon (2020) colocaram o isolamento social como causa de estresse, ansiedade e depressão, quando analisados 208 universitários, no Rio Grande do Sul. Dos 208 participantes, 49\% apresentaram estresse, 33\% ansiedade e 39\% depressão, apontando índices de comprometimento psicológico, no período de pandemia. Em outro estudo realizado com 900 idosos brasileiros, com média de 65 anos de idade revelou que 33\% sentiram-se deprimidos durante a 
pandemia e, ainda, 2,20\% relatou ter tido vontade de se ferir ou de estar morto, o que aponta sérios impactos na saúde mental das pessoas (Pereira-Ávila et al., 2021).

Os impactos psicológicos causados pela pandemia de COVID-19 não foram sentidos apenas pelas pessoas em estado de isolamento social, uma vez que trabalhadores da saúde se mostraram bastante apreensivos e temerosos quanto à exposição ocupacional, frente aos primeiros casos da doença, demonstrados num estudo realizado no interior do Estado do Amazonas (Portugal et al., 2020).

O estilo de vida das pessoas e seus sentimentos são abordados neste estudo, de maneira que é possível dimensionar o que as pessoas sentem no decorrer da pandemia de COVID-19. Mesmo em grupos de pessoas que praticam atividades relaxantes como a Yoga, desenvolveram estresse, ansiedade e depressão, como aponta Correa et al. (2020). Contudo, a prática contínua e o maior tempo de prática semanal, minimiza os efeitos de problemas psicológicos.

Em Minas Gerais, um estudo com 700 pessoas analisou a relação entre pandemia e ganho de peso. O resultado apontou 54\% dos participantes sofreram aumento no peso corporal. Além disso, foram relatados sentimentos de ansiedade em $14,18 \%$, incerteza quanto ao futuro em $13,46 \%$, preocupação em $12,54 \%$ e angústia em $8,04 \%$, sendo estes os sentimentos mais relevantes para a análise (Verticchio, F. R.; Verticchio, N. D. M., 2020).

A autopercepção é um aspecto importante para avaliar a qualidade da saúde mental das pessoas e foi abordada de forma ampla, expondo os sentimentos e o histórico de problemas adquiridos antes da pandemia de COVID-19. Outros fatores como a perda do emprego e diminuição drástica da renda familiar foram enfatizados por Lima et al. (2021), como potencializadores de tristeza, ansiedade, nervosismo e alterações do sono durante a pandemia, a partir da pesquisa envolvendo 45.160 pessoas brasileiras.

O sofrimento psíquico externalizado a partir de situações marcantes perfazem o estudo de Rafael et al. (2021), quando analisou a prevalência e fatores associados em 477 componentes de uma comunidade acadêmica de Enfermagem. Foi evidenciado que sentimentos de solidão, ambiente familiar ruim e histórico de violência familiar estiveram associados ao sofrimento psíquico durante a pandemia.

\section{Conclusão}

A exploração da literatura, realizada neste estudo, permitiu elucidar as questões relacionadas aos impactos psicológicos causados pela pandemia de COVID-19, além de exibir as principais mudanças no estilo de vida das pessoas e seus sentimentos em relação ao atual cenário, como também destacar a autopercepção em relação à sua saúde mental.

Ficou clara a forte relação entre o isolamento social e o desenvolvimento de problemas como ansiedade, depressão e estresse, principalmente em mulheres, uma vez que estas foram as que mais mantiveram-se em confinamento. É evidente que o medo, a incerteza, a tristeza e a preocupação com o adoecer pessoal e familiar são sentimentos que afloraram durante a pandemia. Houve, ainda, uma diminuição drástica na prática de atividades físicas, aumento no consumo de alimentos industrializados, aumento do alcoolismo e tabagismo, bem como alterações no sono.

Entre os homens, a maior preocupação girou em torno do desemprego, da renda familiar, dos conflitos já vividos no lar e na dissolução dos relacionamentos. Dessa forma, pôde-se observar que há muito o que explorar sobre as questões inerentes à saúde mental, de modo que a partir do conhecimento das facetas da mente humana permita o acompanhamento estruturado daqueles que sofrem com problemas psicológicos e transtornos mentais.

\section{Referências}

Barros, M. B. A. et al. (2020). Relato de tristeza/depressão, nervosismo/ansiedade e problemas de sono na população adulta brasileira durante a pandemia de COVID-19. Epidemiologia e Serviços de Saúde, 29, e2020427. 
Bezerra, C. B. et al. (2020) Impacto psicossocial do isolamento durante pandemia de covid-19 na população brasileira: análise transversal preliminar. Saúde e Sociedade, 29, e200412.

Carvalho, L. de S. et al. (2020). O impacto do isolamento social na vida das pessoas no período da pandemia da COVID-19. Research, Society and Development, 9(7), e998975273-e998975273.

Casas, D. G. et al. (2020). COVID-19 y su asociación con síntomas depresivos en población española. Revista Habanera de Ciencias Médicas, 19(5)

Côrrea, C. A. et al. (2020). Níveis de estresse, ansiedade, depressão e fatores associados durante a pandemia de COVID-19 em praticantes de Yoga. Revista Brasileira de Atividade Física \& Saúde, 25, 1-7.

Donida, G. C. C. et al. (2021). Impacto do distanciamento social na saúde mental em tempos de pandemia da COVID-19. Brazilian Journal of Health Review, 4(2), 9201-9218.

Verticchio, F. R.; Verticchio, N. D. M. (2020). Os impactos do isolamento social sobre as mudanças no comportamento alimentar e ganho de peso durante a pandemia do COVID-19 em Belo Horizonte e região metropolitana, Estado de Minas Gerais, Brasil. Research, Society and Development, 9(9), e460997206e460997206.

Duan, L.; Zhu, G. (2020). Psychological interventions for people affected by the COVID-19 epidemic. The Lancet Psychiatry, 7(4), 300-302.

Duarte, M. Q. et al. (2020). COVID-19 e os impactos na saúde mental: uma amostra do Rio Grande do Sul, Brasil. Ciência \& Saúde Coletiva, 25, 3401-3411.

Eidman, L. et al. (2020). Bienestar emocional, psicológico y social en adultos argentinos en contexto de pandemia por covid-19. Psychologia. Avances de la Disciplina, 14(2), 69-80, 2020.

Esteves, C. S.; Oliveira, C. R.; Argimon, I. I. L. (2021). Social distancing: prevalence of depressive, anxiety, and stress symptoms among Brazilian students during the COVID-19 pandemic. Frontiers in Public Health, 8, 923.

Ferreira, D. C. S. et al. (2020). Intolerance of uncertainty and mental health in Brazil during the Covid-19 pandemic. Suma Psicológica, 27(1), 62-69.

Gaygisiz, Ü. et al. (2012). Individual differences in behavioral reactions to H1N1 during a later stage of the epidemic. Journal of infection and public health, $5(1), 9-21$.

Gould, M. S. F.; Diaz, G. C.; Vargas, M. A. R. (2021). Impacto sobre la salud mental durante la pandemia COVID 19 en Paraguay. Revista Virtual de la Sociedad Paraguaya de Medicina Interna, p. 61-68.

Herrera, J. J. P. et al. (2020). Impacto psicosocial de la pandemia por COVID-19 en adultos de Buenos Aires. Rev Argent Cardiol; 88:417-4.

Johnson, M. C.; Saletti-Cuesta, L.; Tumas, N. (2020). Emociones, preocupaciones y reflexiones frente a la pandemia del COVID-19 en Argentina. Ciência \& Saúde Coletiva, 25, 2447-2456.

Lima, M. G. et al. (2021). Associação das condições sociais e econômicas com a incidência dos problemas com o sono durante a pandemia de COVID19. Cadernos de Saúde Pública, v. 37.

Lima, R. C. (2020). Distanciamento e isolamento sociais pela Covid-19 no Brasil: impactos na saúde mental. Physis: Revista de Saúde Coletiva, v. 30, p. e300214.

Lin, E. C. L.; Peng, Y. C.; Tsai, J. C. H. (2010). Lessons learned from the anti-SARS quarantine experience in a hospital-based fever screening station in Taiwan. American journal of infection control, 38(4), 302-307.

Maia, B. R.s; Dias, P. C. (2020). Ansiedade, depressão e estresse em estudantes universitários: o impacto da COVID-19. Estudos de Psicologia (Campinas), v. 37.

Malta, D. C. et al. (2020). Distanciamento social, sentimento de tristeza e estilos de vida da população brasileira durante a pandemia de COVID-19. Saúde em Debate.

Oliveira, J. R., Rocha, R. O. \& Abreu, A. K. M. (2020). A pandemia intramuros:(in) comunicabilidade de mulheres encarceradas em João Pessoa-PB. Revista Interdisciplinar em Cultura e Sociedade. 6(2), 120-41

OMS, Organização Mundial de Saúde. (2021). Panorama Global do Coronavírus.

OPAS, Organização Pan-Americana de Saúde. (2021). Histórico da pandemia de COVID-19.

Ornell, F. et al. (2020). "Pandemic fear" and COVID-19: mental health burden and strategies. Brazilian Journal of Psychiatry, 42(3), $232-235$.

Pereira, Á. L.; Pazos, M. E.; Tögel, M. (2021). Efectos del confinamiento social, preventivo y obligatorio sobre la salud física y psíquica de los comodorenses. Podium. Revista de Ciencia y Tecnología en la Cultura Física, 16(1), 100-113.

Pereira-Ávila, F. M.V. et al. (2021). Fatores associados aos sintomas de depressão entre idosos durante a pandemia da COVID-19. Texto \& ContextoEnfermagem, v. 30.

Portugal, J. K. A. et al. (2020). Percepção do impacto emocional da equipe de enfermagem diante da pandemia de COVID-19: relato de experiência. Revista Eletrônica Acervo Saúde, (46), e3794-e3794.

Prieto-Molinari, D. E. et al. (2020). Depresión y ansiedad durante el aislamiento obligatorio por el COVID-19 en Lima Metropolitana. Liberabit, 26(2). 
Research, Society and Development, v. 10, n. 11, e195101118546, 2021

(CC BY 4.0) | ISSN 2525-3409 | DOI: http://dx.doi.org/10.33448/rsd-v10i11.18546

Qiu, J. et al. (2020). A nationwide survey of psychological distress among Chinese people in the COVID-19 epidemic: implications and policy recommendations. General psychiatry, 33(2).

Rafael, R. M. R. et al. (2021). Sofrimento psíquico na pandemia de COVID-19: prevalência e fatores associados em uma faculdade de enfermagem. Revista Brasileira de Enfermagem, v. 74.

Santos, G. C. et al. (2020). Covid-19 nas prisões: efeitos da pandemia sobre a saúde mental de mulheres privadas de liberdade. Rev. baiana enferm, p. e38235e38235.

Schmidt, B. et al. (2020). Impactos na Saúde Mental e Intervenções Psicológicas Diante da Pandemia do Novo Coronavírus (COVID-19).

SILVA, W. A. D. et al. (2020). Covid-19 no Brasil: estresse como preditor da depressão.

SOUSA, A. R. de et al. (2021). COVID-19 pandemic decrease men's mental health: background and consequence analysis. Jornal Brasileiro de Psiquiatria.

SOUZA, A. S. R. et al. (2021). Factors associated with stress, anxiety, and depression during social distancing in Brazil. Revista de Saúde Pública, v. 55.

Souza, M. T.; SILVA, M. D.; Carvalho, R. (2010). Revisão integrativa: o que é e como fazer. Einstein (São Paulo), 8(1), $102-106$.

Szwarcwald, C. L. et al. (2021). Fatores que afetam a autopercepção de saúde dos brasileiros durante a pandemia da COVID-19. Cadernos de Saúde Pública, 37(3).

WANG, C. et al. (2020). A longitudinal study on the mental health of general population during the COVID-19 epidemic in China. Brain, behavior, and immunity, 87, 40-48.

Yánez, R. J. V. et al. (2021). Estrés percibido asociado a la pandemia por COVID-19 en la ciudad de Guayaquil, Ecuador. Boletín de Malariología y Salud Ambiental, 61(1), 38-46. 